\title{
Cryo-Planing Vs Freeze Fracture: Sample Preparation for Cryo-HRSEM
}

\author{
Irene Y.-T. Chang ${ }^{1}$, Derk Joester ${ }^{1}$ \\ ${ }^{1 .}$ Materials Science and Engineering, Northwestern University, Evanston, IL 60208, USA
}

Cryo-SEM is particularly efficient at revealing the ultrastructure of biological systems in a near-tonative state and at nanometer resolution. Freeze fracture is conveniently used to prepare interior surfaces of frozen-hydrated samples, but the random nature of fracture does not ensure the passage of the fracture plane through the regions of interest $[1,2]$. The resultant surface is rough, which can make interpreting delicate structural features in the sample difficult. To address these issues, we developed the cryo triple ion gun milling (CryoTIGM) technique. High pressure-frozen samples were trimmed using a custombuilt cryo-saw to expose a sample edge, which was brought into contact with a milling mask. Three broad $\mathrm{Ar}^{+}$beams were aimed at the sample edge, and removed materials above the mask to create a cross-section in the sample at the level of the mask. In this manner, large areas in frozen-hydrated samples were cryo-planed in only a few hours. Cryo-planed samples were subsequently freeze-etched and coated with Pt to increase contrast.

We evaluated sample preparation by CryoTIGM against freeze fracture for three biological systems, yeast cell suspensions, mouse liver biopsies, and suspensions of whole sea urchin embryos. The fracture plane frequently occurs between the leaflets of lipid bilayer membranes in freeze-fractured samples, such as the plasma membrane and the nuclear membrane. While this process reveals intra-membranous structures (e.g. nuclear pore complexes), structures inside organelles, for instance the cristae of the inner membrane of mitochondria, cannot be accessed reliably (Fig. 1A, C). In contrast, surfaces cryo-planed using CryoTIGM are very smooth and membranous compartments are always revealed in cross section (Fig. 1B, D). Moreover, morphological information of membranous structures in the out-of-plane direction becomes available. For example, nuclear pores appear as discontinuities in the nuclear membrane in ion-milled samples (Fig. 1D).

Freeze fractured sample surfaces are expectedly rough and provide topographical information about the 3D arrangement. However, this at times complicates interpretation. This shortcoming is exemplified in a comparison of freeze-fractured and CryoTIGM-prepared sea urchin embryo samples (Fig. 2). While both techniques show the ectoderm, the enveloping hyaline layer, and the overall packing of ectodermal cells (Fig. 2A, B), tight contacts among neighboring ectodermal cells, suggesting the presence of intercellular junctions, can be identified only after CryoTIGM (Fig. 2B) Membrane tethers extended from ectodermal cells to the hyaline layer are now visible. The hyaline layer is resolved to consist of two layers, with the space between them occupied by some kind of vesicles or granules. In turns of image resolution and contrast, however, we observe both techniques capable of providing cellular and organellar details at high resolutions and with good contrast. Fundamental components, such as the nucleus, mitochondria, the endoplasmic reticulum, and the Golgi apparatus, can be visualized via both methods.

In conclusion, cryo-planing of frozen-hydrated samples by CryoTIGM is a convenient way to prepare very large, smooth surfaces for subsequent analysis by cryo-HRSEM (and possibly other cryogenic imaging techniques). The ultrastructure of cells and tissues is well preserved and can be imaged at high resolution and with good contrast. Given the large area and high quality of the surfaces, the relatively fast sample preparation, and the excellent preservation of ultrastructural features, CryoTIGM is a 
valuable addition to the toolbox that includes freeze-fracture, cryo-FIB-SEM, and cryo-EM.

References:

[1] Studer, Humbel and Chiquet, Histochemistry and Cell Biology 130 (2008), p. 877.

[2] Gilkey and Staehelin, Journal of Electron Microscopy Technique 3 (1986), p. 177.

[3] The authors acknowledge funding from the NSF Major Research Instrumentation program (NSF

MRI-1229693), the Northwestern University Materials Research Center (DMR-1121262), and the NSF

Biomaterials program (DMR-1106208).

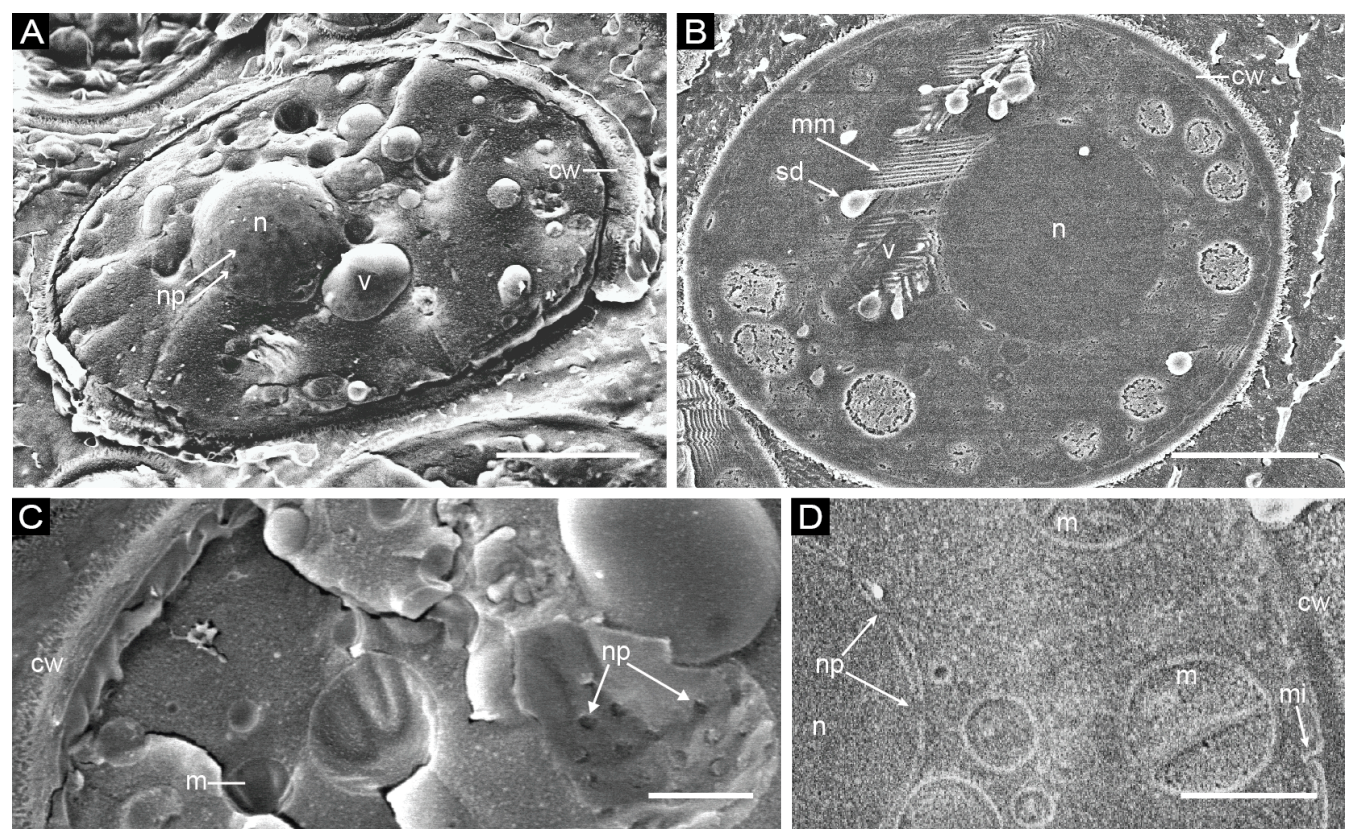

Figure 1. Cryo-SEM images of yeast cell suspensions prepared by freeze fracture (A, C) or CryoTIGM (B, D). cw: cell wall; m: mitochondria; mi: membrane invagination; mm: milling marks; n: nucleus; np: nuclear pore; sd: surface deposits; v: vacuole. Scale bars represent $1.5 \mu \mathrm{m}$ in A, B and $500 \mathrm{~nm}$ in C, D.
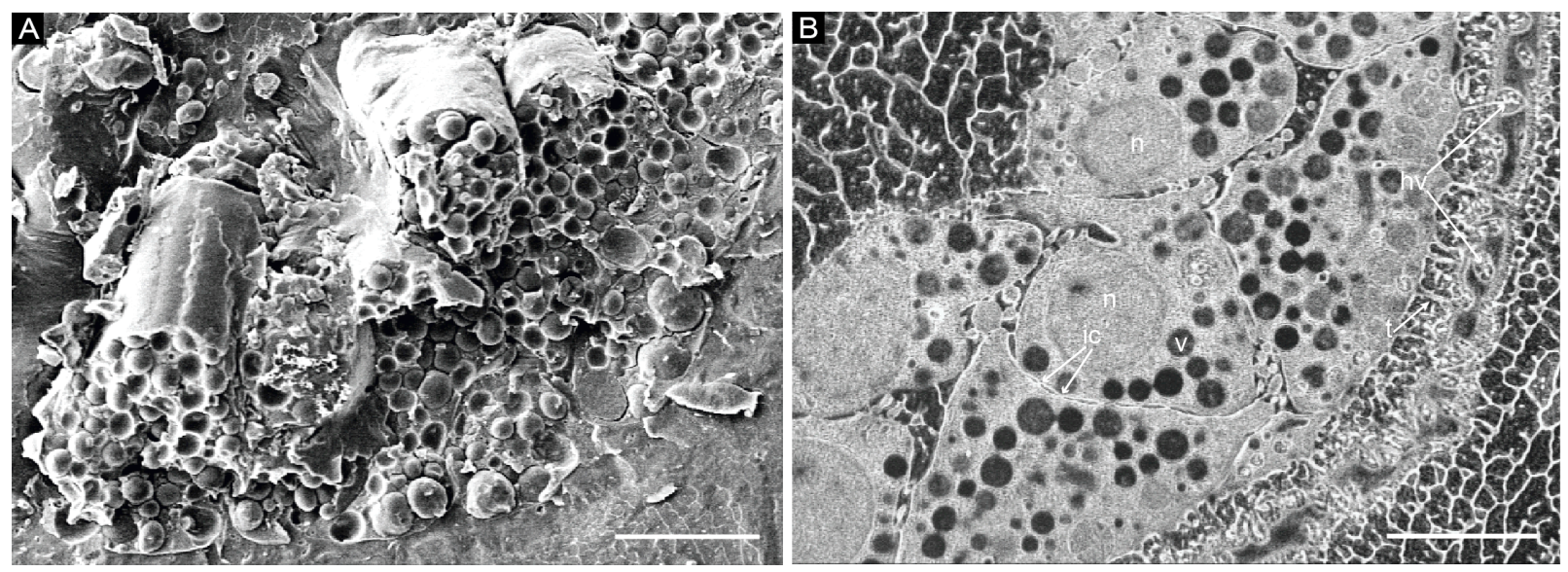

Figure 2. Cryo-SEM images of sea urchin embryos prepared by freeze fracture (A) and CryoTIGM (B), showing part of the thickened vegetal plate. hl: hyaline layer; hv: vesicles within the hyaline layer; ic: intercellular contacts; n: nucleus; t: tethers; v: cytoplasmic vesicle. Scale bars represent $5 \mu \mathrm{m}$. 